\title{
DZK-Stellungnahme zu Tuberkulose, BCG und COVID-19*
}

\author{
( Offizielle Bezeichnung der Erkrankung durch das neuartige Coronavirus SARS-CoV-2, \\ severe acute respiratory syndrome coronavirus 2)
}

\section{DZK Recommendations for Tuberculosis, BCG and COVID-19 in Germany}

\author{
(Official Abbreviation of Coronavirus Disease 2019 Caused by the New Coronavirus \\ SARS-CoV-2)
}

\author{
Autoren \\ N. Schönfeld ${ }^{1,8}$, P. Witte ${ }^{1,9}$, T. Bauer ${ }^{1,7,8}$ \\ Institute \\ 1 Deutsches Zentralkomitee zur Bekämpfung der \\ Tuberkulose e. V. (DZK) \\ 2 Gesundheitsamt Dresden, Dresden \\ 3 LungenClinic Grosshansdorf, Airway Disease Center \\ North (ARCN), Großhansdorf \\ 4 Max-Planck-Institut für Infektionsbiologie, Berlin \\ 5 Bundesministerium für Gesundheit, Berlin \\ 6 Klinik Donaustauf, Universitätsklinikum Regensburg, \\ Regensburg \\ 7 Deutsche Gesellschaft für Pneumologie e. V. (DGP) \\ 8 Helios Klinikum Emil von Behring, Lungenklinik \\ Heckeshorn, Berlin \\ 9 Institut für Krankenhaushygiene der Mühlenkreiskliniken, \\ Universitätsklinikum Johannes-Wesling Minden
}

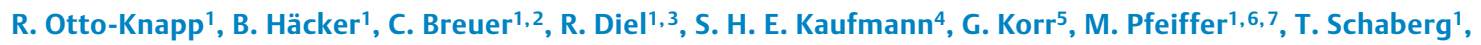

Bibliografie

DOI https://doi.org/10.1055/a-1199-6721 |

Pneumologie 2020; 74: 412-416

(c) Georg Thieme Verlag KG Stuttgart · New York

ISSN 0934-8387

Korrespondenzadresse

Dr. med. Ralf Otto-Knapp, Deutsches Zentralkomitee zur Bekämpfung der Tuberkulose e. V. (DZK), Walterhöferstr. 11, Haus Q, 14165 Berlin info@dzk-tuberkulose.de
Weltweit wurden für das Jahr 2018 von der Weltgesundheitsorganisation (WHO) 1,45 Millionen Todesfälle bei 10 Millionen Tuberkuloseerkrankungen geschätzt [1]. Etwa 4000 Menschen versterben demnach jeden Tag trotz verfügbarer Therapie an Tuberkulose, auch weil schwer therapierbare Medikamentenresistenzen vielerorts ein zunehmendes Problem darstellen. V.a. in Hochprävalenzländern für Tuberkulose kann es während der SARS-CoV-2-Pandemie zu einer Verschlechterung der Fallfindung und der Versorgungssituation von Tuberkulosepatientinnen und -patienten kommen [2]. Eine Modellierungsstudie der Stop TB Partnership kommt zu der Einschätzung, dass zwischen 2020 und 2025 zusätzliche 1,4 Millionen Tuberkulose-Todesfälle entstehen könnten, wenn ein 3-monatiger „Lockdown“ mit einer 10-monatigen Phase der Wiedereinführung der Tuberkuloseversorgung angenommen wird [3]. International wird daher dringend dazu aufgerufen, die Tuberkuloseversorgung auch während der SARS-CoV-2-Pandemie aufrecht zu erhalten. Die weltweiten finanziellen und personellen Ressourcen zur Bekämpfung der Tuberkuloseepidemie müssen er- halten bleiben, um das angestrebte Ziel der Eliminierung nicht zu gefährden [4-6].

Mit einer Inzidenz von 5,8 Neuerkrankungen pro 100000 Einwohnern im Jahr 2019 [7] zählt Deutschland zu den Niedriginzidenzländern mit guten finanziellen und strukturellen Ressourcen im Gesundheitssystem. Versorgungsprobleme für Tuberkulosepatientinnen und -patienten werden in Deutschland auch unter den derzeitigen Pandemie-Bedingungen nicht erwartet. Dennoch können Menschen, die eine Tuberkulosebehandlung erhalten oder abgeschlossen haben oder bei denen eine latente Infektion mit Mycobacterium tuberculosis festgestellt wurde, verständlicherweise durch diese Situation verunsichert sein. Als Hilfestellung für die Beratung von Tuberkulosepatientinnen und -patienten werden daher im Folgenden einige häufig auftretende Fragen zur Tuberkulose und der aktuellen Coronavirus-Pandemie beantwortet. 
Stellt eine vollständig behandelte Tuberkuloseerkrankung ein Risiko für einen schweren Verlauf bei einer Infektion mit SARS-CoV-2 dar?

Zum Zeitpunkt der Erstellung dieses Dokuments existieren nur wenige wissenschaftliche Daten zum Risikoprofil von Menschen mit überstandener Tuberkulose (TB). Vermutlich ist nach einer erfolgreich behandelten TB ohne resultierende Folgeschäden weder die Wahrscheinlichkeit erhöht, eine SARS-CoV-2-Infektion zu bekommen, noch einen schweren Verlauf zu entwickeln. Komorbiditäten und Alter sind allerdings auch in diesem Zusammenhang entscheidende Einflussfaktoren [8].

V.a. in Ländern mit vielen Tuberkulosefällen werden jedoch - meist bedingt durch einen späten Beginn einer nicht immer optimalen Therapie bereits fortgeschrittener Tuberkulosen häufiger Folgeschäden der Lunge wie Bronchiektasen oder obstruktive Lungenerkrankungen (v.a. COPD) beobachtet [911], die im Fall einer Infektion mit SARS-CoV-2 den Verlauf verkomplizieren können. Über die Häufigkeit pulmonaler Folgeerkrankungen nach einer durchgemachten Tuberkulose existieren für Deutschland keine Daten. Sollte eine Folgeerkrankung der Lunge entstanden sein, so können die Risiken für einen schwereren Verlauf im Fall einer SARS-CoV-2-Infektion erhöht sein [12]. Sie orientieren sich wahrscheinlich an Art und Ausmaß der entstandenen Folgeerkrankung [13]. Sollten nach einer Tuberkulosetherapie pulmonale Beschwerden zurückbleiben, muss eine Folgeerkrankung der Lunge ausgeschlossen werden. Eine COPD kann bspw. das Risiko für einen schweren COVID-19-Verlauf erhöhen [14, 15]. Die nationalen Empfehlungen zu Hygienemaßnahmen sollten unbedingt eingehalten werden, um das Infektionsrisiko möglichst gering zu halten. Eine häusliche Quarantäne wird alleine auf der Basis der Grunderkrankung nicht empfohlen. Die adäquate Therapie der Lungenerkrankung trägt zur Stabilisierung bei und kann die Infektanfälligkeit reduzieren. Neben dem Verzicht auf das Rauchen werden eine Pneumokokkenimpfung [13] und eine Grippeschutzimpfung empfohlen. Für weitere Informationen zu Lungenerkrankungen und COVID-19 verweisen wir auf die Stellungnahmen der Deutschen Gesellschaft für Pneumologie [16].

\section{Haben Menschen, die aktuell wegen einer Tuberkuloseerkrankung behandelt werden, ein erhöhtes Risiko für einen schweren Verlauf bei einer Infektion mit SARS-CoV-2?}

Zur Risikobewertung bei Menschen, die während der Tuberkulosetherapie eine SARS-CoV-2-Infektion durchmachen, gibt es keine ausreichenden Daten. In einer Beschreibung von 3 Fällen und einer bislang nur vorveröffentlichten Fall-Kontroll-Studie mit geringer Patientenzahl jeweils aus China wird ein erhöhtes Risiko für einen schweren Krankheitsverlauf vermutet $[17,18]$. Für eine heterogene internationale Kohorte (SARS-CoV-2+Tuberkuloseerkrankung $n=42$; SARS-CoV-2+Folgeschäden nach überstandener Tuberkulose $n=7$ ) wurde eine Case Fatality
Rate von 12,3\% beschrieben, wobei 5 der 6 Verstorbenen älter als 60 Jahre waren und an mindestens einer Begleiterkrankung litten. Die Autoren können aus den bislang vorliegenden Daten keinen kausalen Zusammenhang zwischen Tuberkuloseerkrankung und Mortalität ableiten [8]. Eine weitere Kohorte $(n=20)$ hospitalisierter Patienten mit COVID-19 und Tuberkuloseerkrankung in der Lombardei, Italien, beschreibt 3-4 Wochen nach COVID-19-Diagnose eine Case Fatality Rate von 5\% (1/20 Patienten). Bei der überwiegenden Zahl (19/20) der im Durchschnitt jüngeren Patienten (Median age 39 Jahre) zeigte sich ein benigner klinischer Verlauf mit nur mäßiger Beeinflussung durch die zusätzliche Infektion mit Covid-19 [19]. Es handelt sich aufgrund der geringen Patientenzahlen lediglich um Hinweise, die in Studien mit höherer Fallzahl überprüft werden müssen, bevor Empfehlungen daraus abgeleitet werden können.

Ein erhöhtes Risiko für einen schweren COVID-19-Verlauf durch eine Tuberkuloseerkrankung kann zum jetzigen Zeitpunkt weder belegt noch ausgeschlossen werden. Folgezustände nach durchgemachter Tuberkulose sowie Begleiterkrankungen müssen beachtet werden, da sie zu einem erhöhten Risiko beitragen können. Sollten weitere wissenschaftliche Daten veröffentlicht werden, die eine Änderung der Risikoeinschätzung notwendig machen, wird diese Stellungnahme zeitnah aktualisiert und auf der DZK-Internetseite (www.dzk-tuberkulose.de) verfügbar gemacht. Ein Kompendium der aktuellen Tuberkulose/COVID-19-Studien ist auf den Internetseiten der WHO zu finden (www.who.int/teams/global-tuberculosis-programme/covid-19/compendium).

\section{Tuberkulosebehandlung und Infektion mit SARS-CoV-2}

Um eine Tuberkulosetherapie erfolgreich durchführen zu können, ist es auch unter den aktuell möglicherweise erschwerten Bedingungen wichtig, alle notwendigen Arzttermine wahrzunehmen, um Therapieunterbrechungen zu vermeiden [4]. Vielerorts hat die Coronavirus-Pandemie Umstrukturierungen im Gesundheitssystem zur Folge. Ein negativer Einfluss auf die Versorgungsqualität während der Tuberkulosetherapie [20] und auf die Therapie von Begleiterkrankungen [21] sollte unbedingt vermieden werden.

- Derzeit existiert keine antivirale Standardtherapie für SARSCoV-2. Im Fall von individuellen Therapieversuchen muss jedoch eine Prüfung auf mögliche Wechselwirkungen erfolgen. Medikamenten-Versorgungsengpässe durch eingeschränkte Lieferketten sollten durch eine vorausschauende Therapieplanung vermieden werden.

- Die behandelnden Ärztinnen und Ärzte sollten Tuberkulosepatientinnen und -patienten darüber informieren, dass jeder Kontrolltermin im Rahmen der Therapie wie geplant wahrgenommen werden muss. Auch ungeplante Arztbesuche, bspw. bei Medikamentenunverträglichkeiten oder -nebenwirkungen, müssen jederzeit ohne Verzögerung möglich sein.

- Die soziale Unterstützung von Tuberkulosepatientinnen und -patienten kann Einfluss auf die Therapietreue und auf den Erfolg der Tuberkulosetherapie haben [7, 22]. Notwendige Kontaktbeschränkungen, bspw. die Quarantäne nach Kon- 
takt zu einem SARS-CoV-2-infizierten Menschen, sollten sich nicht negativ auf die Tuberkulosetherapie auswirken. Die vorhandenen Möglichkeiten zur psychosozialen Unterstützung sollten besprochen und wenn möglich ergänzt werden.

- Die Verfügbarkeit von spezialisierten Helfern, z. B. Sozialarbeitern der betreuenden Gesundheitsämter oder Pflegern ambulanter Pflegedienste zur Tablettenausgabe, kann unter den aktuellen Pandemie-Bedingungen eingeschränkt sein. Mögliche Alternativen zur Therapiebegleitung können digitale Lösungen bieten [4]. Eine in Deutschland etablierte und empfohlene Plattform zur Unterstützung durch digitale Systeme existiert nicht, daher müssen individuelle Lösungen gefunden werden. Zur multilingualen Patienteninformation hat sich die Applikation ExplainTB (www.explaintb.org) bewährt.

- Verzögerungen bei der Diagnostik der Tuberkulose durch die zeitweise Überlastung der ärztlichen Versorgungsangebote sollten vermieden werden, um einen verzögerten Therapiebeginn bei schon fortgeschrittener Erkrankung zu verhindern. Verzögerungen bei Fallfindung und Therapie können zu vermehrten Übertragungen führen. Im Rahmen von Ausgangsbeschränkungen und häuslicher Quarantäne sind zudem vermehrte Übertragungen innerhalb einer Wohneinheit möglich.

\section{Ist eine latente Tuberkuloseinfektion ein Risiko bei einer Infektion mit SARS-CoV-2?}

Die latente Infektion mit Mycobacterium tuberculosis ist nicht mit einer Tuberkuloseerkrankung gleichzusetzen. Hinter dem Begriff der latenten Infektion verbirgt sich ein Spektrum von Zuständen nach Kontakt zum Tuberkuloseerreger. Das Spektrum reicht dabei von der rein immunologischen Auseinandersetzung mit dem Erreger ohne Persistenz von Tuberkulosebakterien über den Verbleib kleiner Erregermengen unter immunologischer Kontrolle bis hin zur in Entwicklung befindlichen Tuberkuloseerkrankung [23, 24]. Die verfügbaren Nachweisverfahren (Tuberkulinhauttest und Interferon-Gamma Release Assays [IGRAs]) können nur die Auseinandersetzung des Immunsystems mit dem Erreger anzeigen. Ob Tuberkuloseerreger im Körper verblieben sind und ob diese reaktivieren also durch Vermehrung zu einer manifesten Erkrankung führen -, können die Tests nicht voraussagen. Unter diesen Voraussetzungen wird eine einheitliche Risikobewertung für diese heterogene Gruppe schwer zu treffen sein.

Ein allgemein erhöhtes Risiko für virale Atemwegserkrankungen wird für Menschen mit latenter Tuberkuloseinfektion nicht angenommen. Bis aussagekräftige Daten zur latenten Tuberkuloseinfektion und SARS-CoV-2 zur Verfügung stehen, ist keine Aussage zu speziellen Risiken in dieser Gruppe möglich.

\section{Kann ich mich mithilfe einer Tuberkulose- Impfung (Bacille Calmette-Guérin; BCG) vor einer Infektion mit SARS-CoV-2 schützen?}

Ein möglicher heterologer Schutz-Effekt der BCG-Impfung wird diskutiert und in verschiedenen Ländern in Studien geprüft. Bis Studienergebnisse vorliegen, die einen Nutzen für die BCGImpfung bei SARS-CoV-2-Infektionen belegen, wird der Einsatz der BCG-Impfung zum Schutz vor SARS-CoV-2-Infektionen oder schweren Verläufen von COVID-19 von der WHO [25] ebenso wie von der International Union Against Tuberculosis and Lung Disease [26] ausdrücklich nicht empfohlen. Die BCG-Impfung soll ausschließlich indikationsgerecht bei Neugeborenen in Tuberkulose-Hochinzidenzländern eingesetzt werden. Eine zusätzliche Verknappung der weltweit vorhandenen Impfdosen kann die Kindersterblichkeit in vielen Ländern erhöhen und muss unbedingt vermieden werden $[27,28]$. Dieser Empfehlung schließt sich das DZK an. In Deutschland wird eine BCG-Impfung seit 1998 grundsätzlich von der Ständigen Impfkommission (STIKO) am Robert Koch-Institut nicht mehr empfohlen.

Die Vermutung einer möglichen Schutzwirkung wurde aufgrund der Beobachtung aufgestellt, dass nach der weltweiten Einführung der BCG-Impfung in einigen Ländern eine Verringerung der Sterblichkeit von Kindern gesehen wurde und dieser Effekt über den Schutz gegen Tuberkulose hinausging. Experimentelle und klinische Daten zeigten, dass eine BCG-Impfung zumindest für einen begrenzten Zeitraum unspezifische Effekte auf das Immunsystem hat, die die Abwehr von viralen und möglicherweise von bakteriellen Erregern verbessern können [29]. Die Annahme, dass die BCG-Impfung bei SARS-CoV-2-Infektionen schützt, beruht bislang nur auf epidemiologischen Daten. Die Autoren einer Studie vermuteten einen positiven Einfluss der BCG-Impfung auf die Mortalität und Morbidität von SARSCoV-2-Infektionen im Rahmen der aktuellen Coronavirus-Pandemie [30] (Details im DZK-Newsletter im Internet: www.dzktuberkulose.de/aktuelles/newsletter und in dieser Ausgabe der Pneumologie). Andere Studien lieferten widersprüchliche epidemiologische Daten zu dieser Hypothese [31, 32].

Die epidemiologischen Studiendaten liefern allerdings keine ausreichende Evidenz, um einen Zusammenhang zwischen der BCG-Impfung und einer Reduktion der COVID-19-bedingten Mortalität zu belegen. Zudem werden einige Schwächen im Studiendesign diskutiert [33]. Dennoch wurde eine Hypothese geschaffen, die in Wissenschaft und Medien intensiv diskutiert wird [34-36]. Die experimentellen und klinischen Hinweise für unspezifische Effekte der BCG-Impfung auf das Immunsystem führten dazu, dass größere Placebo-kontrollierte Studien an Mitarbeitern im Gesundheitssystem, die einem erhöhten Infektionsrisiko ausgesetzt sind, in den Niederlanden, Australien und Südafrika begonnen wurden (www.clinicaltrials.gov). Diese sollen innerhalb eines Jahres Antworten zu den Fragen generieren, ob die BCG-Impfung einen Einfluss auf die Häufigkeit von COVID-19 oder den Krankheitsverlauf hat. Weitere Studien wurden in verschiedenen anderen Ländern angemeldet (www. clinicaltrials.gov). Auch eine Phase-III-Studie mit einer am MaxPlanck-Institut für Infektionsbiologie in Berlin entwickelten ge- 
netisch modifizierten Variante des BCG-Impfstoffs (VPM1002) wird an verschiedenen deutschen Kliniken getestet. Impfdosen von VPM1002 wären kurzfristig in großer Stückzahl herstellbar [37], da sie nicht in Kulturen gewonnen werden müssen, sondern in Bioreaktoren mit hohem Durchsatz hergestellt werden können. Im Falle einer Wirksamkeit dieses Ansatzes könnte damit eine zusätzliche Verknappung der weltweit verfügbaren BCG-Impfdosen vermieden werden.

\section{BCG-IMPFUNG IN DEUTSCHLAND}

Die BCG-Impfung ist ein attenuierter Lebendimpfstoff, der 1921 erstmals beim Menschen angewendet wurde. In der BRD wurde die Impfung mit BCG generell für alle Neugeborenen auf freiwilliger Basis bis 1975 empfohlen, danach folgten Risiko-bezogene Einschränkungen der Impfindikation. In der DDR wurde die BCG-Impfung in den frühen 1950er-Jahren als Pflichtimpfung bei Kindern eingeführt, die später auf Neugeborene ausgeweitet wurde. Nach der Wiedervereinigung 1990 wurden die Empfehlungen der BRD übernommen. Seit 1998 wird die BCGImpfung in Deutschland nicht mehr empfohlen und ist aktuell nicht verfügbar. Indikationsgerecht werden speziell aufbereitete BCG-Impfstämme in Deutschland nur bei der Immuntherapie des Harnblasenkarzinoms als Blaseninstillation eingesetzt. Im März 2020 erschien ein Rote-Hand-Brief, der auf das Risiko einer systemischen BCG-Infektion nach Blaseninstillation hinweist.

\section{BCG-IMPFUNG WELTWEIT}

Weltweit wird die BCG-Impfung in Regionen mit hoher Tuberkuloseinzidenz (hier definiert als 40 Fälle/100000 Einwohner pro Jahr) für Neugeborene empfohlen [38]. Sie bietet Schutz gegen besonders schwere Verlaufsformen wie die Miliartuberkulose und die tuberkulöse Meningitis, v.a. in den 3 ersten Lebensjahren [39]. Die BCG-Impfung ist mit derzeit knapp 130 Millionen Impfdosen pro Jahr wichtiger Bestandteil nationaler Impfstrategien in Hochinzidenzländern und kann zur Senkung der Kindersterblichkeit beitragen. In den letzten Jahren ist es zu weltweiten Lieferengpässen für den BCG-Impfstoff gekommen [40]. Negative Auswirkungen auf die Kindersterblichkeit in den betroffenen Ländern müssen angenommen werden [25, 26, 39, 41]. In Südafrika zeigte sich einer Studie zufolge bereits ein signifikanter Anstieg der Fallzahlen für tuberkulöse Meningitis im Jahr 2017, der den Studiendaten zufolge auf einen Rückgang der BCGImpfungen zurückzuführen ist [42]. Der Einsatz von BCG zum Schutz vor einer SARS-CoV-2-Infektion außerhalb von Studien ist wissenschaftlich zum jetzigen Zeitpunkt nicht gerechtfertigt. Er könnte aber zu einer weiteren Verknappung des BCG-Impfstoffes führen und weltweit erhöhte Mortalität durch schwere Tuberkulosefälle bei Kindern zur Folge haben [25-28].

\section{Zusammenfassung}

- Die wenigen vorhandenen wissenschaftlichen Daten können zum jetzigen Zeitpunkt zusätzliche Risiken durch eine Tuberkuloseerkrankung für einen schweren COVID-19-Verlauf weder belegen noch ausschließen.

- Die Tuberkuloseversorgung muss auch unter den derzeitigen Pandemie-Bedingungen in vollem Umfang aufrechterhalten werden, um den Erfolg der Tuberkulosetherapie nicht zu gefährden.

- Folgezustände nach überstandener Tuberkuloseerkrankung, insbesondere im Falle einer COPD, können je nach Art und Schwere ein erhöhtes Risiko für einen schweren COVID-19Verlauf bedeuten.

- Für Menschen mit latenter Tuberkuloseinfektion wird allgemein kein erhöhtes Risiko für virale Atemwegserkrankungen angenommen. Zu den Risiken speziell bei SARS-CoV-2-Infektionen gibt es keine ausreichenden Daten.

- Die BCG-Impfung soll ausschließlich indikationsgerecht in Ländern mit hoher Tuberkuloseinzidenz eingesetzt werden. In Deutschland wird sie seit 1998 generell nicht mehr empfohlen.

- Der Einsatz von BCG zum Schutz vor einer SARS-CoV-2-Infektion außerhalb von Studien ist zum jetzigen Zeitpunkt nicht gerechtfertigt. Ein großflächiger Einsatz von BCG würde zur Verknappung des Impfstoffs beitragen und kann gravierende Folgen für die Kindersterblichkeit in Hochinzidenzländern haben.

\section{Danksagung}

Besonderer Dank für die inhaltliche Unterstützung bei der Erstellung des Manuskriptes gilt Barbara Hauer, Robert Koch-Institut, Berlin.

Interessenkonflikt

Prof. Stefan H.E. Kaufmann ist Ko-Erfinder des Impfstoffs VPM1002, der an Vakzinze Projekt Management $\mathrm{GmbH}$ und Serum Institute of India Ltd. lizenziert wurde. Dr. Brit Häcker: finanzielle Unterstützung der Firma Otsuka für den Aufbau einer Datenbank für MDR-Patienten. Dr. Ralf Otto-Knapp hat Honorare der Firmen Novartis, Gilead, Boehringer Ingelheim, Berlin Chemie, Insmed, Astra Zeneca für Vorträge erhalten, die von den genannten Firmen finanziell unterstützt oder organisiert wurden. Die Firmen hatten keinen Einfluss auf die Inhalte der Präsentationen. Prof. Roland Diel hat Honorare der Firmen Bayer, Cepheid, Insmed, Otsuka und Quidel für Vorträge, Beratungstätigkeit oder Forschungsunterstützung erhalten.

Literatur

[1] Global tuberculosis report 2019. Geneva: World Health Organization; 2019. Licence: CC BY-NC-SA 3.0 IGO

[2] Glaziou P. Predicted impact of the COVID-19 pandemic on global tuberculosis deaths in 2020. medRxiv 2020. doi:10.1101/ 2020.04.28.20079582

[3] Stop TB Partnership. The potential impact of the COVID-19 response on tuberculosis in high-burden countries: a modelling analysis. Im Internet (Stand: 09.06.2020): http://www.stoptb.org/covid19.asp

[4] World Health Organization (WHO). Information Note Tuberculosis and COVID-19: Considerations for tuberculosis (TB) care. 2020. Im Internet (Stand: 09.06.2020): https://www.who.int/news-room/de- 
tail/12-05-2020-updated-who-information-note-ensuring-continuity-of-tb-services-during-the-covid-19-pandemic

[5] Adepoju P. Tuberculosis and HIV responses threatened by COVID-19. Lancet HIV 2020; 7: e319-e320

[6] Union warns the COVID-19 pandemic must not divert attention from the needs of children and adolescents in TB-endemic African countries. Im Internet (Stand: 09.05.2020): https://www.theunion.org/ news-centre/news/union-warns-the-covid-19-pandemic-must-notdivert-attention-from-the-needs-of-children-and-adolescents-in-tbendemic-african-countries

[7] Robert Koch-Institut. Epidemiologisches Bulletin 11/2020. 2020. Im Internet (Stand: 09.05.2020): https://www.rki.de/DE/Content/Infekt/ EpidBull/Archiv/2020/11/Tabelle.html

[8] Tadolini M, Codecasa LR, García-García J-M et al. Active tuberculosis, sequelae and COVID-19 co-infection: first cohort of 49 cases. Eur Respir J 2020: 2001398. doi:10.1183/13993003.01398-2020

[9] Ravimohan S, Kornfeld H, Weissman D et al. Tuberculosis and lung damage: from epidemiology to pathophysiology. Eur Respir Rev 2018; 27: 170077. doi:10.1183/16000617.0077-2017

[10] Gupte AN, Paradkar M, Selvaraju S et al. Assessment of lung function in successfully treated tuberculosis reveals high burden of ventilatory defects and COPD. PloS One 2019; 14: e0217289

[11] Lee C-H, Lee M-C, Lin H-H et al. Pulmonary tuberculosis and delay in anti-tuberculous treatment are important risk factors for chronic obstructive pulmonary disease. PloS One 2012; 7: e37978

[12] International Union Against Tuberculosis and Lung Disease. COVID-19 and lung health: Frequently Asked Questions. Im Internet (Stand: 09.05.2020): https://www.theunion.org/news-centre/covid-19/covid-tb-faqs\#five

[13] Deutsche Gesellschaft für Pneumologie (DGP). Stellungnahme - Risikoabschätzung bei Patienten mit chronischen Atemwegs- und Lungenerkrankungen im Rahmen der SARS-CoV-2-Pandemie. 2020. Im Internet (Stand: 09.06.2020): https://pneumologie.de/aktuelles-service/covid-19/

[14] Alqahtani JS, Oyelade T, Aldhahir AM et al. Prevalence, Severity and Mortality associated with COPD and Smoking in patients with COVID19: A Rapid Systematic Review and Meta-Analysis. PloS One 2020; 15 : e0233147

[15] Guo FR. Smoking links to the severity of Covid-19: An update of a meta-analysis.] Med Virol 2020. doi:10.1002/jmv.25967 [Online ahead of print]

[16] Deutsche Gesellschaft für Pneumologie (DGP). COVID-19. Im Internet (Stand: 09.06.2020): https://pneumologie.de/aktuelles-service/covid-19/

[17] He G, Wu J, Shi J et al. COVID-19 in Tuberculosis patients: a report of three cases. J Med Virol 2020. doi:10.1002/jmv.25943

[18] Liu Y, Bi L, Chen Y et al. Active or latent tuberculosis increases susceptibility to COVID-19 and disease severity. medRxiv 2020. doi:10.1101/2020.03.10.20033795

[19] Stochino C, Villa S, Zucchi P et al. Clinical characteristics of COVID-19 and active tuberculosis co-infection in an Italian reference hospital. Eur Respir J 2020: 2001708. doi:10.1183/13993003.01708-2020

[20] Saunders M], Evans CA. COVID-19, tuberculosis, and poverty: preventing a perfect storm. Eur Respir J 2020: 2001348. doi:10.1183/ 13993003.01348-2020

[21] Rosenbaum L. The Untold Toll - The Pandemic's Effects on Patients without Covid-19. N Engl J Med 2020. doi:10.1056/NEJMms2009984

[22] Schaberg T, Bauer T, Brinkmann F et al. [Tuberculosis Guideline for Adults - Guideline for Diagnosis and Treatment of Tuberculosis including LTBI Testing and Treatment of the German Central Committee (DZK) and the German Respiratory Society (DGP)]. Pneumologie 2017; 71: 325-397
[23] Pai M, Behr MA, Dowdy D et al. Tuberculosis. Nat Rev Dis Primer 2016; 2: 16076

[24] Drain PK, Bajema KL, Dowdy D et al. Incipient and Subclinical Tuberculosis: a Clinical Review of Early Stages and Progression of Infection. Clin Microbiol Rev 2018; 31: e00021-18

[25] World Health Organization. Scientific Brief - Bacille Calmette-Guérin (BCG) vaccination and COVID-19. 2020. Im Internet (Stand: 09.05.2020): https://www.who.int/news-room/commentaries/detail/bacille-calmette-gu\%C3\%A9rin-(bcg)-vaccination-and-covid-19

[26] Schaaf HS, du Preez K, Kruger M. Bacille Calmette-Guérin (BCG) vaccine and the COVID-19 pandemic: responsible stewardship is needed. 2020. Im Internet (Stand: 09.05.2020): https://www.theunion.org/ news-centre/news/bcg-vaccine-and-covid-19-responsible-stewardship-is-needed

[27] Marais B], Seddon JA, Detjen AK et al. Interrupted BCG vaccination is a major threat to global child health. Lancet Respir Med 2016; 4: 251253

[28] Kontturi A, Santiago B, Tebruegge $M$ et al. The impact of Bacille Calmette-Guerin shortage on immunisation practice and policies in Europe - A Paediatric Tuberculosis Network European Trials Group (ptbnet) survey. Tuberc 2016; 101: 125-129

[29] Moorlag SJCFM, Arts RJW, van Crevel R et al. Non-specific effects of BCG vaccine on viral infections. Clin Microbiol Infect 2019; 25: 1473-1478

[30] Miller A, Reandelar MJ, Fasciglione K et al. Correlation between universal BCG vaccination policy and reduced morbidity and mortality for COVID-19: an epidemiological study. medRxiv 2020. doi:10.1101/ 2020.03.24.20042937

[31] Berg MK, Yu Q, Salvador CE et al. Mandated Bacillus Calmette-Guérin (BCG) vaccination predicts flattened curves for the spread of COVID19. medRxiv 2020. doi:10.1101/2020.04.05.20054163

[32] Hensel J, McGrail DJ, McAndrews KM et al. Exercising caution in correlating COVID-19 incidence and mortality rates with BCG vaccination policies due to variable rates of SARS CoV-2 testing. medRxiv 2020. doi:10.1101/2020.04.08.20056051

[33] Kumar J, Meena J. Demystifying BCG Vaccine and COVID-19 Relationship. Indian Pediatr 2020; 57: 588-589

[34] Hamiel U, Kozer E, Youngster I. SARS-CoV-2 Rates in BCG-Vaccinated and Unvaccinated Young Adults. JAMA 2020: e208189. doi:10.1001/ jama.2020.8189

[35] O'Neill LA], Netea MG. BCG-induced trained immunity: can it offer protection against COVID-19? Nat Rev Immunol 2020; 20: 335-337. doi:10.1038/s41577-020-0337-y

[36] Redelman-Sidi G. Could BCG be used to protect against COVID-19? Nat Rev Urol 2020; 17: 316-317. doi:10.1038/s41585-020-0325-9

[37] Lawton G. Trials of BCG vaccine will test for covid-19 protection. New Sci 1971 2020; 246: 9. doi:10.1016/S0262-4079(20)30836-8

[38] World Health Organization. BCG vaccines: WHO position paper February 2018. Vaccins BCG: Note de synthèse de l'OMS - Février 2018. Wkly Epidemiol Rec 2018; 93: 73-96

[39] Trunz BB, Fine P, Dye C. Effect of BCG vaccination on childhood tuberculous meningitis and miliary tuberculosis worldwide: a meta-analysis and assessment of cost-effectiveness. Lancet 2006; 367: 11731180

[40] Cernuschi T, Malvolti S, Nickels E et al. Bacillus Calmette-Guérin (BCG) vaccine: A global assessment of demand and supply balance. Vaccine 2018; 36: 498-506

[41] Roy P, Vekemans J, Clark A et al. Potential effect of age of BCG vaccination on global paediatric tuberculosis mortality: a modelling study. Lancet Glob Health 2019; 7: e1655-e1663

[42] du PreezK, Seddon JA, Schaaf HS et al. Global shortages of BCG vaccine and tuberculous meningitis in children. Lancet Glob Health 2019; 7: e28-e29

Hinweis: Der Artikel wurde nachträglich um Angaben zu den Interessenkonflikten ergänzt. 